\title{
Understanding the Assembly Mechanism of Proteins from Monte Carlo Simulations
}

\author{
Stéphane Cuenot ${ }^{*}$, Agata Zykwinska², Sadia Radji³ ${ }^{3}$ Jean-Philippe Bouchara ${ }^{4,5}$ \\ ${ }^{1}$ Institut des Matériaux Jean Rouxel (IMN), Université de Nantes, Nantes, France \\ ${ }^{2}$ IFREMER, Laboratoire Ecosystèmes Microbiens et Molécules Marines pour les Biotechnologies, Nantes, France \\ ${ }^{3}$ IPREM Equipe de Physique et Chimie des Polymères, Université de Pau et des Pays de l'Adour, Pau, France \\ ${ }^{4}$ UNIV Bretagne-Loire, Université d'Angers, Université de Brest, Groupe d'Etude des Interactions Hôte-Pathogène, \\ Angers, France \\ ${ }^{5}$ Laboratoire de Parasitologie-Mycologie, Centre Hospitalier Universitaire, Institut de Biologie en Santé, \\ Angers, France \\ Email: *stephane.cuenot@cnrs-imn.fr
}

How to cite this paper: Cuenot, S., Zykwinska, A., Radji, S. and Bouchara, J.-P. (2017) Understanding the Assembly Mechanism of Proteins from Monte Carlo Simulations. Applied Mathematics, 8, 280-292. https://doi.org/10.4236/am.2017.83023

Received: December 13, 2016 Accepted: February 28, 2017

Published: March 3, 2017

Copyright $\odot 2017$ by authors and Scientific Research Publishing Inc. This work is licensed under the Creative Commons Attribution International License (CC BY 4.0).

http://creativecommons.org/licenses/by/4.0/

\begin{abstract}
Understanding the molecular mechanism of the protein assembly still remains a challenge in the case of many biological systems. In this frame, the mechanism which drives RodA hydrophobins to self-assemble onto the surface of the conidia of the human fungal pathogen Aspergillus fumigatus into highly ordered nanorods known as rodlets, is still unresolved. Here, AFM investigations were combined with Monte Carlo simulations to elucidate how these small amphiphilic proteins self-assemble into tightly packed rodlets and how they are further organized in nanodomains. It becomes that the assembly of RodA hydrophobins into rodlets and their parallel alignment within nanodomains result from their anisotropic properties. Monte Carlo simulations allowed us to confirm that anisotropic interactions between macromolecules are sufficient to drive them to assembly into rodlets prior to nanodomains formation. Better knowledge of the mechanism of hydrophobins assembly into rodlets offers new prospects for the development of novel strategies leading to inhibition of rodlet formation, which should allow more rapid detection of the conidia by the immune system.
\end{abstract}

\section{Keywords}

Protein Assembly, Aspergillus fumigatus, Anisotropic Interactions, Monte Carlo Simulations

\section{Introduction}

The self-assembly of proteins into small-scale complexes is a ubiquitous phe- 
nomenon that is of great importance to biology. For instance, protein aggregation occurs in several diseases such as Alzheimer's [1] and Parkinson's [2] diseases, as well as in type II diabetes [3]. Understanding the mechanism of protein assembly to develop strategies to prevent this phenomenon becomes therefore fundamental. Besides experiments, modeling and numerical simulation methods offer an interesting alternative approach to decipher the pertinent parameters involved into these complex mechanisms of assembly. In this way, Monte Carlo simulations have been applied to get further insight into assembly process of various proteins by using their anisotropic character both in their shape and in their interactions [4]. One of the advantages of Monte Carlo methods concerns the great flexibility to model different kinds of interactions between particles and to easily check their effect on the resulting assembly. Recently, several simulation works have shown that proteins interacting via anisotropic or "patchy" attractions can spontaneously assemble into anisotropic structures [5]. The use of particles with anisotropic interactions (patchy particles) in Monte Carlo simulations represents a reasonable compromise between the complexity of interactions involved in assembly processes and the relative simplicity required for modelling [5] [6]. By playing with several parameters, such as the amplitude of interactions and the number of inter-particle contacts, various self-assembly processes of polymers, colloids and proteins were simulated [5] [6]. In particular, assemblies of peptides and proteins into amyloid fibrils were also obtained by considering strongly anisotropic and highly directional interactions which mimic the cross- $\beta$ spine structure [4] [7]. Such structure favours the assembly of proteins via $\beta$ sheets which confer an important anisotropic character to proteins.

In the present study, Atomic Force Microscopy (AFM) experiments and Monte Carlo simulations were combined to address a biologically pertinent question relative to the formation mechanism of protein nanodomains on the conidial surface of one of the major human pathogens, Aspergillus fumigatus. Indeed, this filamentous fungus represents a huge threat for immunocompromised patients by causing severe and often fatal disseminated infections [8] [9]. This is because of difficulties establishing a prompt diagnosis and of the low efficiency of current systemic antifungals [8] [9]. The initial step in the majority of infectious diseases is the adhesion of pathogenic organisms to host cells and tissues [10]. Being responsible for the attachment to host tissues, the surface of $A$. fumigatus conidia plays a crucial role in the infection and its investigation represents an important concern for the development of new therapeutic strategies. Indeed, like few other filamentous fungi, $A$. fumigatus produces conidia, which are covered by a characteristic layer of parallel and regularly spaced nanorods, called rodlets. Rodlets are composed of amphiphilic proteins, so called hydrophobins and RodA hydrophobins were demonstrated to be responsible for the rodlet structuring on the surface of $A$. fumigatus mature conidia [11] [12]. The rodlet layer serves multiple purposes, including conferring water resistance to conidia for easier dispersion in air currents and enhancing adherence to some hydrophobic surfaces. Aimanianda et al. [13] have recently demonstrated that 
the rodlet layer plays a crucial role in the pathogenicity of $A$. fumigatus since it masks the cell wall components from their recognition by the immune system. Therefore, it appears clearly that rodlets can be identified as an important virulence factor and it becomes crucial to elucidate their formation mechanism. Up to now, the mechanism which drives RodA hydrophobins to assemble into highly ordered rodlet structures has not been fully elucidated. In particular, the organization of hydrophobins into parallel nanorods regularly spaced and the formation of nanodomains covering the whole surface of conidia remain to be determined. In this context, Monte Carlo simulations were devoted here to test a possible mechanism of RodA hydrophobins self-assembly into rodlets covering the surface.

\section{Material and Methods}

\subsection{Fungal Strains}

Experiments were conducted on A. fumigatus reference strain IHEM 18963 (Af293) which was previously used for genome sequencing of $A$. fumigatus and that produces typical, dark blue green powdery colonies on yeast extract-peptonedextrose agar (YPDA).

\subsection{Media, Growth Conditions and Preparation of Conidial Suspensions}

The fungal strain was maintained by weekly passages on YPDA medium (containing in $\mathrm{g} / \mathrm{L}$ : yeast extract, 5; peptone, 10; glucose, 20; and agar, 20) supplemented with chloramphenicol $0.5 \mathrm{~g} / \mathrm{L}$. Conidia from 5 -day-old cultures at $37^{\circ} \mathrm{C}$ were then fixed on glass coverslips previously coated with laminin (Sigma-Aldrich). After incubation for $30 \mathrm{~min}$ at $37^{\circ} \mathrm{C}$ under constant shaking, coverslips were washed in PBS to remove unbound laminin, and then applied directly at the surface of sporulating colonies of the fungus (5-day-old cultures on YPDA). Finally, coverslips were washed in PBS buffer and then dried at room temperature before AFM analysis.

\subsection{Atomic Force Microscopy Measurements}

The surface of $A$. fumigatus conidia was imaged using a NanoWizard ${ }^{\oplus}$ atomic force microscope (AFM, JPK, Germany) operating in intermittent contact mode under ambient conditions. A standard rectangular cantilever (Nanosensors NCLW) was used for imaging, with a free resonance frequency of $165 \mathrm{kHz}$ and a typical spring constant of about $40 \mathrm{~N} / \mathrm{m}$. The radius curvature of the tip was $\sim 10$ $\mathrm{nm}$. The detailed analysis of AFM images were performed using JPK Data Processing software (JPK, Germany).

\section{Results and Discussion}

\subsection{Surface Characterization of $A$. fumigatus Conidia}

Atomic Force Microscopy (AFM) image presented on Figure 1(a) reveals that 
the outer surface of $A$. fumigatus conidia is fully covered by nanodomains composed of parallel and regularly spaced rodlets [14]. The size of nanodomains, composed of at least three rodlets, varied from 120 to $220 \mathrm{~nm}$ in length and from 60 to $100 \mathrm{~nm}$ in width. Although it was previously demonstrated that each rodlet constitutes an assembly of several RodA hydrophobins [11] [12], the mechanism which drives hydrophobins to assemble into highly ordered rodlets and their further structuring into nanodomains has not been elucidated yet. Recently, the detailed structural work performed on similar hydrophobins, namely EAS [15] [16] allowed to generate a molecular model for their self-assembly into a rodlet monolayer [17]. It was shown that EAS hydrophobins are organized in a cross- $\beta$ spine structure with $\beta$-sheets formed from extended strands of the protein segment and hydrogen bounded up and down to the identical macromolecules. Such an amyloid structure has already been proposed for other amyloid-forming proteins and peptides [18] [19].

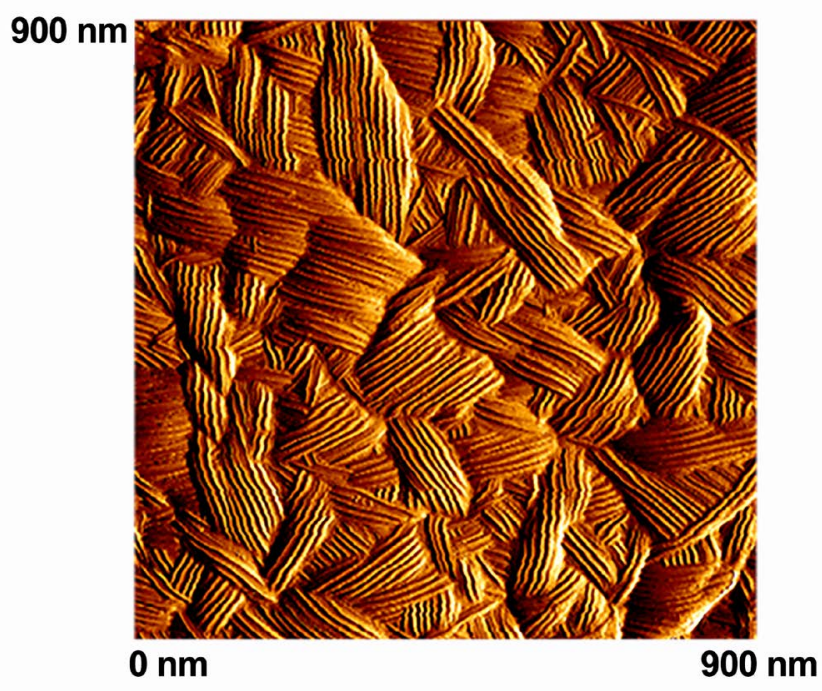

(a)

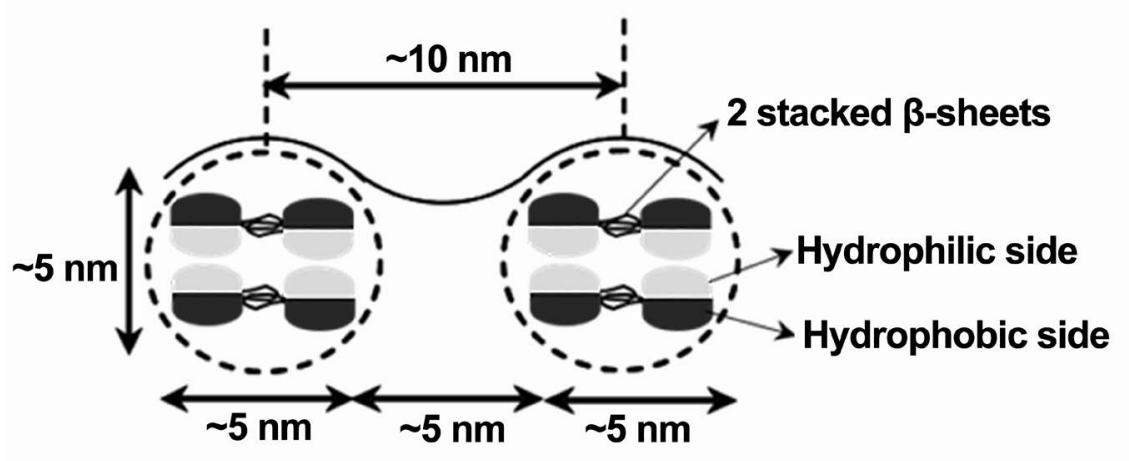

(b)

Figure 1. (a) AFM amplitude image of rodlets organized within nanodomains on the surface of a single $A$. fumigatus conidium; (b) Schematic representation of rodlets crosssection where each rodlet is constituted by a bilayer of two RodAhydrophobin dimers (the geometric dimensions are extracted from the AFM image). 
Rodlet forming hydrophobins were shown to be globular proteins of similar diameters, close to $2.5 \mathrm{~nm}$ [17]. Therefore, taking into account the $A$. fumigatus rodlet thickness of $\sim 5 \mathrm{~nm}$, measured from AFM images, and the diameter of a single hydrophobin of $\sim 2.5 \mathrm{~nm}$, it comes out that $A$. fumigatus rodlets are most likely organized in bilayers. In addition, the distance between the tops of two successive rodlets, i.e. the rodlet inter-distance of $\sim 10 \mathrm{~nm}$ measured from AFM images suggests both that each rodlet is spaced out from its neighbouring rodlet by a distance of $\sim 5 \mathrm{~nm}$ and that the section of each rodlet is approximately a circle of $\sim 5 \mathrm{nmin}$ diameter. One possible configuration to fulfil such a rodlet dimension is the Rod A. assembly into bilayers composed of superimposed dimers stabilized by $\beta$-sheet stacks between anti-parallel molecules (Figure 1(b)). Since the outer surface of $A$. fumigatus conidia is uniformly hydrophobic, as shown by adhesion measurements performed by AFM [20], hydrophobic domains of RodA dimers point outward, while their hydrophilic parts point inward [21].

During conidia formation, hydrophobins are secreted by the fungus into its surrounding environment (e.g. hydrated growth medium). The amphiphilic nature drives hydrophobins to self-assembly into rodlets that pack tightly to form amphipathic layers at different hydrophobic-hydrophilic interfaces (e.g. airgrowth medium) [22]. Such interfacial assembly can directly be related to the protein interface concentration through the Gibbs equation [23]. The Gibbs equation implies that the surface tension decreases with the logarithm of the protein concentration, up to reach a constant value for the critical assembly concentration, $C^{*}$ [23] [24]. At this critical concentration, the interface is fully covered by assembled proteins and the surface tension is minimized with the lowest packing energy, corresponding to the stable assembly state. However, at sufficiently high concentration $\left(C>C^{*}\right)$, several proteins have been shown to self-assemble in the bulk solution in small asymmetric assemblies like oligomers [25] [26] [27]. These spontaneous assemblies are strongly dependent on the anisotropic character of proteins both in their shape and in their interactions. Therefore, according to Gibbs equation, at high concentration, RodA hydrophobins may assemble not only at the air-growth medium interface but also in the bulk of the aqueous growth medium. Indeed, the presence of soluble oligomers rich in $\beta$-sheets was already reported in aqueous solutions of SC3 hydrophobins [28] [29]. The fact that very few defects were observed within rodletnanodomains imaged by AFM (Figure 1(a)) indicates that the elementary unit constitutive of rodlets is very small. Indeed, from a topological point of view, to pave a surface with lines without defects (as holes or dislocations) is only possible with small building blocks, such as oligomers. Therefore, RodA hydrophobins start probably to self-assemble in dimers in the surrounding medium through stacked $\beta$-sheets between anti-parallel molecules.

\subsection{Modelling for the Monte Carlo Simulations}

Some research groups have recently studied the peculiar case of phase behaviour of interacting rigid rods on two-dimensional square lattices by Monte Carlo si- 
mulations [30] [31] [32]. In this study, we propose to simulate the assembly of RodA hydrophobins on a surface in the monolayer regime with anisotropic interactions between particles using Monte Carlo method. In our model, each particle, representing a RodAdimer (Figure 1(b)), interacts with its four nearestneighbours through anisotropic interactions modelled by a patchy model [30] [33]. Precisely, each particle possesses two bonding poles on two opposite sides inducing that two patches on different particles can only interact if they overlap. Thus, this anisotropy of the inter-particle interactions allows to mimic the formation of cross- $\beta$ spine structure by favouring the particle alignment. Such patchy models have recently focused an increasing interest for simulations of protein crystallization and self-assembly of particles into chains [33]. Our Monte Carlo simulations would allow to either confirm or reject the assumption that the anisotropy of inter-hydrophobin interactions can control their assembly by constructing a possible scenario of rodlet formation covering the surface. As the RodA hydrophobin assembly takes spontaneously place in the surrounding medium during the conidia formation, the use of dynamic methods is not necessarily required to describe the surface organization of mature conidia [7].

The surface of $A$. fumigatus is modelled as a two-dimensional square lattice of $150 \times 150$ adsorptive sites where periodic boundary conditions were applied in both horizontal and vertical directions. These conditions allow 1) to simulate an infinite surface where all sites are equivalent avoiding the border effects and 2) to represent the spherical geometry of $A$. fumigatus conidia. Particles representing RodAdimers are adsorbed on the surface with two allowed orientations along the principal axis of the square lattice. Each site of the lattice is characterized by a three-state variable where the value 0 corresponds to an empty site, and the values \pm 1 represent the vertical and horizontal orientations, respectively. In order to represent the properties of a monolayer regime, each lattice site can only be single-occupied.

The following Monte Carlo procedure was used to simulate the adsorption process. Starting from an initial state where all sites of the square lattice are empty, a particle is adsorbed on one of the lattice sites at random and its orientation is also chosen randomly (horizontal or vertical orientation with probability $1 / 2$ each). Then, another particle with its orientation chosen as random is adsorbed on an empty site of the lattice chosen as random. If there is no neighbouring particle, the orientation of the new particle is chosen at random. If it is not the case, the orientation of the particle is determined by calculating the Hamiltonian of the system by considering the interactions with its four nearestneighbours. The Hamiltonian of the system is described in Equation 1

$$
H=-J \sum_{<k, l>}\left|\vec{S}_{i, j} \vec{r}_{i j-k l}\right|\left|\vec{S}_{k, l} \vec{r}_{i j-k l}\right|
$$

where $J$, is the interaction strength between two nearest-neighbour particles (kept constant in the simulations); $\langle k, b$, represents a sum over the nearest-neighbours sites identified by the row $\mathrm{k}$ and column 1 on the two-dimensional square lattice; $\vec{S}_{i, j}$, is the occupation and orientation vector of site identi- 
fied by the row $i$ and column $j$ (similar definitions are used for the occupation and orientation vector of nearest-neighbours sites identified by the row $k$ and column $I ; \vec{r}_{i j-k l}$, corresponds to the inter-particles vector connecting the sites (i, $j)$ and $(k, I)$.

The interaction strength between two nearest particles depends both on their relative orientations and on their orientations relative to the inter-particles vector (Equation (1)). Precisely, the bond energy is $-J$ if two particles are nearest neighbours on the lattice and both axes are parallel to the lattice vector connecting them (particles aligned), and is zero otherwise. Thus, for a given configuration, the energy is directly proportional to the number of shared bonds between particles. In the Monte Carlo procedure, the Hamiltonian is first calculated for the trial configuration with the random orientation, $H_{\text {initial }}$. Then, an attempt is made to change the orientation state and the corresponding Hamiltonian is calculated, $H_{\text {final }}$. The orientation change is determined with the probability given by the standard Metropolis acceptance criterion (Equation (2)):

$$
P=\min \left[1, \exp \left(-\Delta H / k_{B} T\right)\right]
$$

where $\Delta H=H_{\text {final }}-H_{\text {initial }}$, is the difference between the Hamiltonians of the final and initial states. If $\Delta H<0$, the energy of the system is minimized, the orientation change is accepted and the process is repeated by choosing another site. If $\Delta H>0$, the Boltzmann weight is compared to a random number comprised between 0 and 1 . If this random number is lower than the Boltzmann weight, the orientation change is accepted; otherwise this change is rejected. At each time step, a new particle is added on the lattice and its orientation is either chosen at random if there is no particle in its neighbouring or chosen to minimize the total energy by considering the interactions with the nearest particles. It is important to note that the configurational entropy is implicitly taken into account in such Monte Carlo simulations with a jointly optimization of the total energy and configurational entropy of the system [31]. The simulations were performed at constant temperature $\mathrm{T}$, but the aggregation kinetics could also be simulated using Monte Carlo dynamics for different values of $k_{B} T$ (where $k_{B}$ is the Boltzmann constant) [7]. Monte Carlo simulations using a deposition-evaporation algorithm of particles could also be an alternative to our model but the final result should not be significantly different [34].

\subsection{Monte Carlo Simulations of Rodletnano Domain Formation}

Results of Monte Carlo simulations are shown on Figure 2 through four snapshots corresponding to different coverage ratio $(\chi)$ of adsorbed particles on the surface. For low coverage ratio $(\chi=25 \%)$, the first adsorbed particles are either isolated on the square lattice or they initiate the particle assembly into short lines (Figure 2(a)). For $\chi=50 \%$, by adding more adsorbed particles on the lattice, the rod formation appears more clearly with longer linear assemblies (Figure 2(b)). The first rod formed acts as a nucleation site to induce the formation of other rods parallel to the first one (repulsive interactions between rods). These longer 


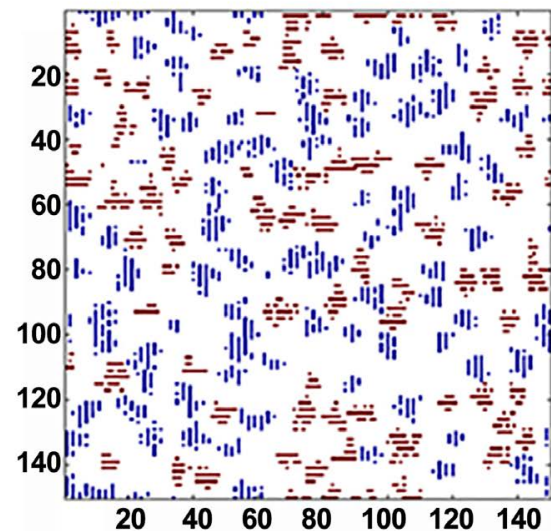

(a)

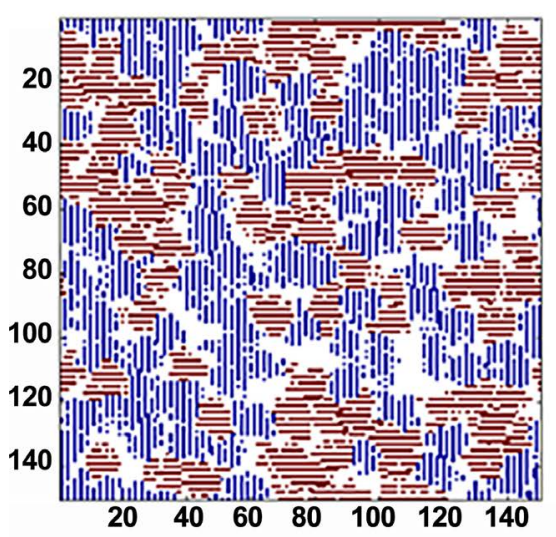

(c)

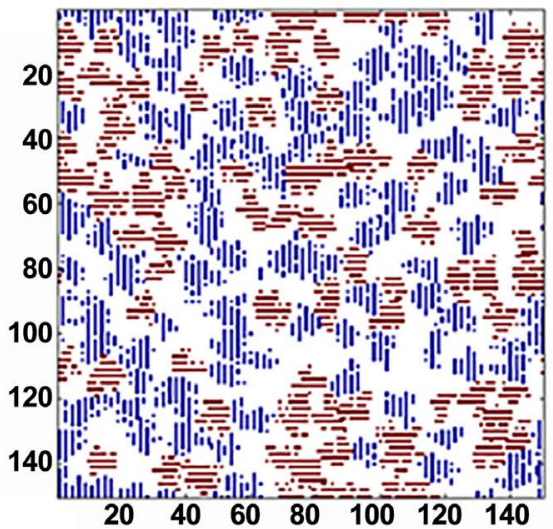

(b)

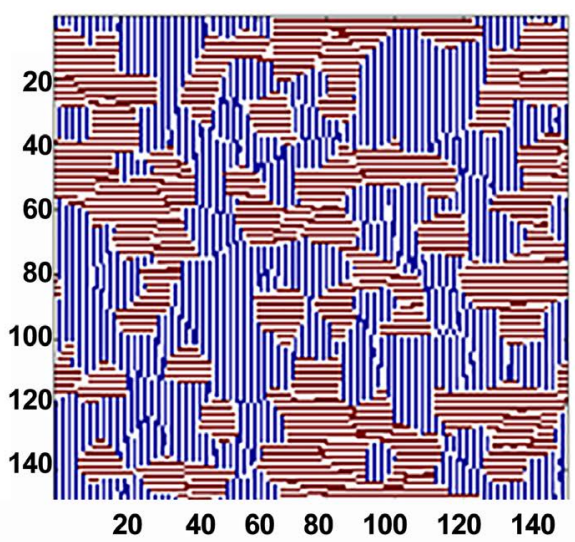

(d)

Figure 2. Snapshots of Monte Carlo simulations showing the domains formed from particles assembled into lines (which model RodA dimers assembly into rodlets before the formation of nanodomains) for different surface coverage ratios (a) $25 \%$, (b) $50 \%$, (c) $75 \%$, (d) $100 \%$.

rods correspond to the assembly of RodA hydrophobin dimers into rodlets through anisotropic interactions between stacked $\beta$-sheets, which conduct to a final linear cross- $\beta$ spine structure. By increasing the coverage ratio up to $75 \%$, the simulations show that the linear assemblies generate the formation of domains, which contain some parallel lines not totally complete (Figure 2(c)). When $\chi$ reaches $100 \%$, the lattice is fully covered by domains that are composed of several parallel rods (Figure 2(d)). The boundaries of domains correspond to breaking symmetry coming from the intersection of lines of adjacent domains.

Based on the minimization of the total energy of the system with an underlying maximization of configurational entropy, our Monte Carlo simulations reveal that by taking only into account anisotropic interactions between the particles on a square lattice, several domains of parallel lines resulting from the linear assembly of particles were formed. The good agreement obtained between the AFM image of the protein assembly covering the surface and the final simulation, where the lines within domains modelled well the rodlets observed (Figure 1 and Figure 2), shows that a sufficient condition for the self-assembly of 
hydrophobins into rodlets is to consider anisotropic interactions between hydrophobins. The anisotropic interactions used in our patchy model possess a repulsive part (end-to-end dimers orientation) and an attractive part (side-to-side dimers orientation) modelling the interactions between hydrophobins through stacked $\beta$-sheets. However, it is not necessary to define precisely the nature of these anisotropic interactions in our simulations. Other simulations describing explicitly the anisotropic character of interactions with attractive/repulsive electrostatic interactions could lead to similar results [31] [32].

From Figure 2, Monte Carlo simulations reveal a possible scenario to fully cover the surface of rodlet domains with 1) the formation of rodlets from the assembly of RodAhydrophobin dimers via the cross- $\beta$ spine structure and 2) the subsequent formation of nanodomains. From Monte Carlo simulations, more detailed information can be obtained on the assembly mechanism by following the evolution of the rodlet number and rodlet length with the amount of adsorbed particles. In Figure 3(a), four distinct parts can be clearly distinguished on the curve of the average number of rodlets plotted as a function of the coverage ratio. In the first part, a linear increase in the average rodlet number up to 130 was observed for $\chi=0.8 \%$. This comes from the fact that each new particle added on the lattice generates the formation of a new rodlet. For $\chi$ from $0.8 \%$ to $40 \%$, the average number of rodlets increased strongly from 130 to 1200 . Indeed, new particles are still added on the lattice, which leads to the formation of new rodlets. However, in this part of the curve, new particles may also associate (i.e. assemble) to existing rodlets. For $\chi$ from $40 \%$ to $70 \%$, a slight increase in the average rodlet number was noticed (up to 1500), which suggests that new particles contribute most likely to the increase in length of rodlets already formed rather than to formation of new rodlets. In the last part of the curve, when the lattice

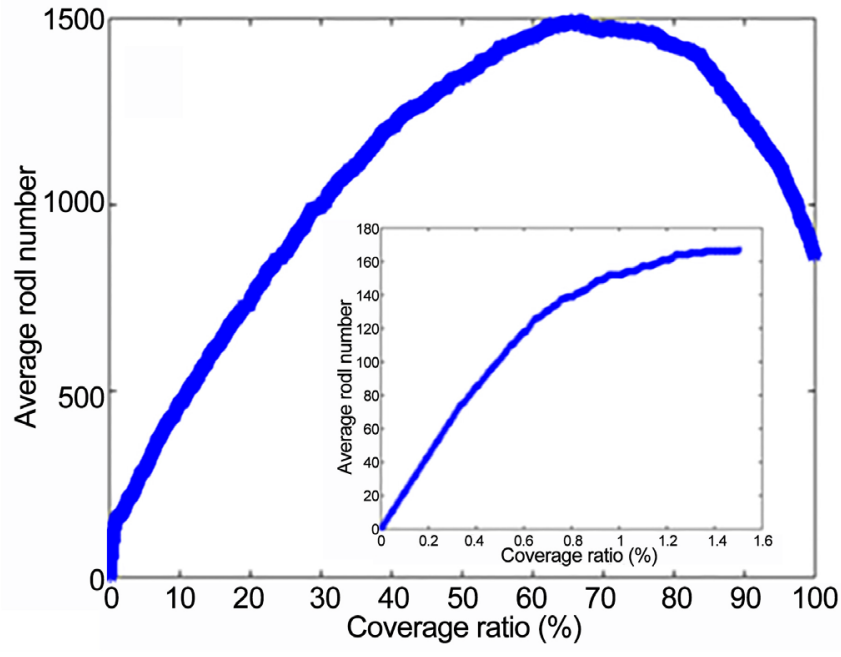

(a)

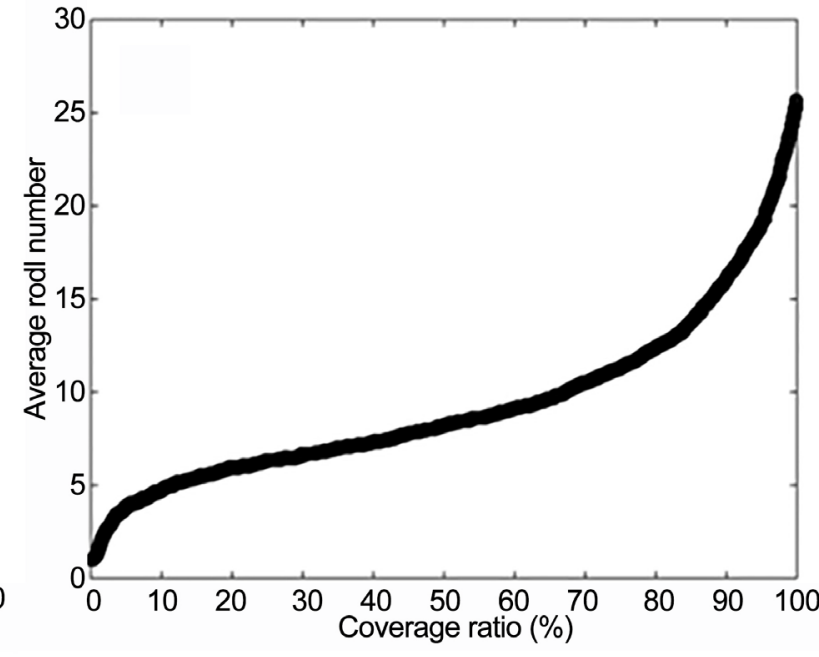

(b)

Figure 3. (a) Evolution of the average number of rodlets formed in function of the surface coverage ratio, deduced from Monte Carlo simulation. In the inset, the linear dependence observed for the first adsorbed particles indicating that they systematically initiate the formation of new rodlets; (b) Evolution of the average length of rodlets formed in function of the surface coverage ratio, obtained from Monte Carlo simulation. 
becomes completely covered ( $\chi$ from $70 \%$ to $100 \%$ ), the average number of rodlets significantly decreased down to 850 . It is most likely that new added particles contribute to connect rodlets already formed (Figure 2(c)), which decreases the average number of rodlets on the lattice. From Monte Carlo simulations, the evolution of the average length of rodlets can also be followed in function of the coverage ratio (Figure $3(\mathrm{~b})$ ). Three parts are clearly visible. For $\chi$ up to $5 \%$, the average rodlet length increased rapidly to get rodlets constituted of 4 units. In the second part, when $\chi$ increased from $5 \%$ to $85 \%$, new added particles contribute to a continuous increase in the average rodlet length up to 13 units. Finally, the average length of rodlets strongly increased, leading to rodlets composed of 25 units, when the lattice was almost completely covered ( $\chi$ from 85\% to $100 \%)$. Indeed, as already suggested, the addition of new particles led to association of existing rodlets, thus resulting in important increase in the rodlet length.

The first monolayer of RodAhydrophobin dimers assembled into rodletsvia anisotropic interactions between stacked $\beta$-sheets on the conidia surface is most likely continuously completed by free dimers from growth medium. These free dimers spontaneously interact through their hydrophilic sides with the monolayer already assembled to decrease the monolayer surface energy. Finally, the formed bilayer constitutes the real rodlet as schematically shown on Figure 1(b).

\section{Conclusion}

In the present work, Monte Carlo simulations were used to support AFM investigations of the conidial surface in the human opportunistic pathogen A. fumigatus to understand the mechanism that leads RodA hydrophobins to self-assemble into rodlets. These small proteins, which possess an important anisotropic character via their interactions through stacked $\beta$-sheets, are ideal candidates to be modelled. Monte Carlo simulations allowed showing that anisotropic interactions between RodA hydrophobindimers (which are pre-assembled in the growth medium) are sufficient to drive them to assembly into parallel rodlets prior to the formation of nanodomains. These interactions possess an attractive part for side-to-side RodA hydrophobin dimers orientation modelling the interactions between hydrophobins through stacked $\beta$-sheets and a repulsive part for end-toend dimers orientation. Such Monte Carlo simulations can be used as a complementary tool of experiments, offering a great support in the understanding of assembly mechanisms. Therefore, the complexity of mechanisms governing the assembly of peptides, colloids and proteins can be overcome.

\section{Acknowledgements}

The authors acknowledge the "Region des Pays de la Loire" for financial support in the frame of "Myco-AFM" research program.

\section{References}

[1] Chiti, F. and Dobson, C.M. (2006) Protein Misfolding, Functional Amyloid, and 
Human Disease. Annual Review of Biochemistry, 75, 333-366. https://doi.org/10.1146/annurev.biochem.75.101304.123901

[2] Uversky, V.N. and Eliezer, D. (2009) Biophysics of Parkinson's Disease: Structure and Aggregation of $\alpha$-Synuclein. Current Protein \& Peptide Science, 10, 483-499. https://doi.org/10.2174/138920309789351921

[3] Andersson, A., Bohman, S., Borg, L.A.H., Paulsson, J.F., Schultz, S.W., Westermark, G.T. and Westermark, P. (2008) Amyloid Deposition in Transplanted Human Pancreatic Islets: A Conceivable Cause of Their Long-Term Failure. Experimental Diabetes Research, 2008, Article ID: 562985. https://doi.org/10.1155/2008/562985

[4] Auer, S., Dobson, C.M., Vendruscolo, M. and Maritan, A. (2008) Self-Templated Nucleation in Peptide and Protein Aggregation. Physical Review Letters, 101, Article ID: 258101. https://doi.org/10.1103/physrevlett.101.258101

[5] Bianchi, E., Blaak, R. and Likos, C.N. (2011) Patchy Colloids: State of the Art and Perspectives. Physical Chemistry Chemical Physics, 13, 6397-6410. https://doi.org/10.1039/c0cp02296a

[6] Doppelbauer, G., Bianchi, E. and Kahl, G. (2010) Self-Assembly Scenarios of Patchy Colloidal Particles in Two Dimensions. Journal of Physics: Condensed Matter, 22, Article ID: 104105. https://doi.org/10.1088/0953-8984/22/10/104105

[7] Irbäck, A., Jonsson, S.A., Linnemann, N., Linse, B. and Wallin, S. (2013) Aggregate Geometry in Amyloid Fibril Nucleation. Physical Review Letters, 110, Article ID: 058101. https://doi.org/10.1103/physrevlett.110.058101

[8] Latgé, J.P. (1999) Aspergillus fumigatus and Aspergillosis. Clinical Microbiology Reviews, 12, 310-350.

[9] Nivoix, Y., Velten, M., Letscher-Bru, V., Moghaddam, A., Natarajan-Amé, S., Fohrer, C., Lioure, B., Bilger, K., Lutun, P., Marcellin, L., Launoy, A., Bergerat, J.P. and Herbrecht, R. (2008) Factors Associated with Overall and Attributable Mortality in Invasive Aspergillosis. Clinical Infectious Diseases, 47, 1176-1184. https://doi.org/10.1086/592255

[10] Tronchin, G., Pihet, M., Lopes-Bezerra, L.M. and Bouchara, J.-P. (2008) Adherence Mechanisms in Human Pathogenic Fungi. Medical Mycology, 46, 749-772. https://doi.org/10.1080/13693780802206435

[11] Thau, N., Monod, M., Crestani, B., Rolland, C., Tronchin, G., Latgé, J.-P. and Paris, S. (1994) Rodletless Mutants of Aspergillus fumigates. Infection and Immunity, 62, 4380-4388.

[12] Paris, S., Debeaupuis, J.-P., Crameri, R., Carey, M., Charlès, F., Prévost, M.C., Schmitt, C., Philippe, B. and Latgé, J.-P. (2003) Conidial Hydrophobins of Aspergillus fumigatus. Applied and Environmental Microbiology, 69, 1581-1588. https://doi.org/10.1128/AEM.69.3.1581-1588.2003

[13] Aimanianda, V., Bayry, J., Bozza, S., Kniemeyer, O., Perruccio, K., RamuluElluru, S., Clavaud, C., Paris, S., Brakhage, A.A., Kaveri, S.V., Romani, L. and Latgé, J.-P. (2009) Surface Hydrophobin Prevents Immune Recognition of Airborne Fungal Spores. Nature, 460, 1117-1121. https://doi.org/10.1038/nature08264

[14] Dague, E., Alsteens, D., Latgé, J.-P., Verbelen, C., Raze, D., Baulard, A.R. and Dufrêne, Y.F. (2007) Chemical Force Microscopy of Single Live Cells. Nano Letters, 7, 3026-3030. https://doi.org/10.1021/nl071476k

[15] Kwan, A.H., Winefield, R.D., Sunde, M., Matthews, J.M., Haverkamp, R.G., Templeton, M.D. and Mackay, J.P. (2006) Structural Basis for Rodlet Assembly in Fungal Hydrophobins. Proceedings of the National Academy of Sciences of the United States of America, 103, 3621-3626. https://doi.org/10.1073/pnas.0505704103 
[16] Kwan, A.H., Macindoe, I., Vukašin, P.V., Morris, V.M., Kass, I., Gupte, R., Mark, A.E., Templeton, M.D., Mackay, J.P. and Sunde, M. (2008) The Cys3-Cys4 Loop of the Hydrophobin EAS Is Not Required for the Formation and Surface Activity. Journal of Molecular Biology, 382, 708-720. https://doi.org/10.1016/j.jmb.2008.07.034

[17] Macindoe, I., Kwan, A.H., Ren, Q., Morris, V.K., Yang, W., Mackay, J.P. and Sunde, M. (2012) Self-Assembly of Functional, Amphipathic Amyloid Monolayers by the Fungal Hydrophobin EAS. Proceedings of the National Academy of Sciences of the United States of America, 109, 804-811. https://doi.org/10.1073/pnas.1114052109

[18] Nelson, R., Sawaya, M.R., Balbirnie, M., Madsen, A.O., Riekel, C., Grothe, R. and Eisenberg, D. (2005) Structure of the Cross-Beta Spine of Amyloid-Like Fibrils. Nature, 435, 773-778. https://doi.org/10.1038/nature03680

[19] Goldschmidt, L., Teng, P.K., Riek, R. and Eisenberg, D. (2010) Identifying the Amylome, Proteins Capable of Forming Amyloid-Like Fibrils. Proceedings of the National Academy of Sciences of the United States of America, 107, 3487-3492. https://doi.org/10.1073/pnas.0915166107

[20] Dague, E., Alsteens, D., Latgé, J.-P. and Dufrêne, Y.F. (2008) High-Resolution Cell Surface Dynamics of Germinating Aspergillus fumigatus Conidia. Biophysical Journal, 94, 656-660. https://doi.org/10.1529/biophysj.107.116491

[21] Zykwinska, A., Pihet, M., Radji, S., Bouchara, J.-P. and Cuenot, S. (2014) SelfAssembly of Proteins into a Three-Dimensional Multilayer System: Investigation of the Surface of the Human Fungal Pathogen Aspergillus fumigatus. Biochimica et Biophysica Acta, 1844, 1137-1144. https://doi.org/10.1016/j.bbapap.2014.03.001

[22] Wösten, H.A.B., Schuren, F.H.J. and Wessels, J.G.H. (1994) Interfacial Self-Assembly of a Hydrophobin into an Amphipathic Protein Membrane Mediates Fungal Attachment to Hydrophobic Surfaces. EMBO Journal, 13, 5848-5854.

[23] Gibbs, J.W. (1931) The Collected Works of J. W. Gibbs. Longmans, Green, New York, Vol. 1, 219.

[24] Adamson, A.W. and Gast, A.P. (1997) Physical Chemistry of Surfaces. 4th Edition, John Wiley, New York.

[25] Fung, S.Y., Keyes, C., Duhamel, J. and Chen, P. (2003) Concentration Effect on the Aggregation of a Self-Assembling Oligopeptide. Biophysical Journal, 85, 537-548. https://doi.org/10.1016/S0006-3495(03)74498-1

[26] Liu, X.Y. (2000) Molecular Modelling for Non-Ideal Mixing of Amphiphilic Molecules. Langmuir, 18, 14-25. https://doi.org/10.1021/la0105329

[27] Sipe, J.D. and Cohen, J.D. (2000) Review: History of the Amyloid Fibril. Journal of Structural Biology, 130, 88-98. https://doi.org/10.1006/jsbi.2000.4221

[28] Wang, X., Graveland-Bikker, J.F., de Kruif, C.G. and Robillard, G.T. (2004) Oligomerization of Hydrophobin SC3 in Solution: From Soluble State to Self-Assembly. Protein Science, 13, 810-821. https://doi.org/10.1110/ps.03367304

[29] Zykwinska, A., Guillemette, T., Bouchara, J.-P. and Cuenot, S. (2014) Spontaneous Self-Assembly of SC3 Hydrophobins into Nanorods in Aqueous Solution. Biochimica et Biophysica Acta, 1844, 1231-1237. https://doi.org/10.1016/j.bbapap.2014.04.003

[30] Tavares, J.M., Holder, B. and Telo da Gama, M.M. (2009) Structure and Phase Diagram of Self-Assembled Rigid Rods: Equilibrium Polydispersity and Nematic Ordering in Two Dimensions. Physical Review E, 79, Article ID: 021505. https://doi.org/10.1103/PhysRevE.79.021505

[31] Longone, P., Linares, D.H. and Ramirez-Pastor, A.J. (2010) Critical Behaviour of 
Attractive Rigid Rods on Two-Dimensional Lattices. Journal of Chemical Physics, 132, Article ID: 184701. https://doi.org/10.1063/1.3424775

[32] Ghosh, A. and Dhar, D. (2007) On the Orientational Ordering of Long Rods on a Lattice. Europhysics Letters, 78, 20003. https://doi.org/10.1209/0295-5075/78/20003

[33] Doye, J.P.K., Louis, A.A., Lin, I.-C., Allen, L.R., Noya, E.G., Wilber, A.W., Kok, H.C. and Lyus, R. (2007) Controlling Crystallization and Its Absence: Proteins, Colloids and Patchy Models. Physical Chemistry Chemical Physics, 9, 2197-2205. https://doi.org/10.1039/b614955c

[34] Schwen, E.M., Mazilu, I. and Mazilu, D.A. (2015) A Stochastic Model of Particle Deposition and Evaporation for Ionic Self-Assembly of Thin Films. Journal of Physics, 574, Article ID: 012043. https://doi.org/10.1088/1742-6596/574/1/012043

Submit or recommend next manuscript to SCIRP and we will provide best service for you:

Accepting pre-submission inquiries through Email, Facebook, LinkedIn, Twitter, etc. A wide selection of journals (inclusive of 9 subjects, more than 200 journals)

Providing 24-hour high-quality service

User-friendly online submission system

Fair and swift peer-review system

Efficient typesetting and proofreading procedure

Display of the result of downloads and visits, as well as the number of cited articles Maximum dissemination of your research work

Submit your manuscript at: http://papersubmission.scirp.org/

Or contact am@scirp.org 